\title{
RESEARCH
}

Open Access

\section{DNA methylation, transcriptome and genetic copy number signatures of diffuse cerebral WHO grade II/III gliomas resolve cancer heterogeneity and development}

H. Binder ${ }^{1 *+}$ (D) E. Willscher ${ }^{1 \dagger}$, H. Loeffler-Wirth ${ }^{1}$, L. Hopp ${ }^{1}$, D. T. W. Jones ${ }^{2,3}$, S. M. Pfister ${ }^{2,4,5}$, M. Kreuz ${ }^{6}$, D. Gramatzki ${ }^{7}$, E. Fortenbacher', B. Hentschel ${ }^{6}$, M. Tatagiba ${ }^{8}$, U. Herrlinger ${ }^{9}$, H. Vatter ${ }^{9}$, J. Matschke $^{10}$, M. Westphal ${ }^{11}$, D. Krex ${ }^{12}$,

G. Schackert ${ }^{12}$, J. C. Tonn ${ }^{13}$, U. Schlegel ${ }^{14}$, H.-J. Steiger ${ }^{15}$, W. Wick ${ }^{16,17}$, R. G. Weber ${ }^{18}$, M. Weller ${ }^{7 \dagger}$ and M. Loeffler ${ }^{6+}$

\begin{abstract}
Background: Diffuse lower WHO grade II and III gliomas (LGG) are slowly progressing brain tumors, many of which eventually transform into a more aggressive type. LGG is characterized by widespread genetic and transcriptional heterogeneity, yet little is known about the heterogeneity of the DNA methylome, its function in tumor biology, coupling with the transcriptome and tumor microenvironment and its possible impact for tumor development.

Methods: We here present novel DNA methylation data of an LGG-cohort collected in the German Glioma Network containing about 85\% isocitrate dehydrogenase (IDH) mutated tumors and performed a combined bioinformatics analysis using patient-matched genome and transcriptome data.

Results: Stratification of LGG based on gene expression and DNA-methylation provided four consensus subtypes. We characterized them in terms of genetic alterations, functional context, cellular composition, tumor microenvironment and their possible impact for treatment resistance and prognosis. Glioma with astrocytoma-resembling phenotypes constitute the largest fraction of nearly $60 \%$. They revealed largest diversity and were divided into four expression and three methylation groups which only partly match each other thus reflecting largely decoupled expression and methylation patterns. We identified a novel G-protein coupled receptor and a cancer-related 'keratinization' methylation signature in in addition to the glioma-CpG island methylator phenotype (G-CIMP) signature. These different signatures overlap and combine in various ways giving rise to diverse methylation and expression patterns that shape the glioma phenotypes. The decrease of global methylation in astrocytoma-like LGG associates with higher WHO grade, age at diagnosis and inferior prognosis. We found analogies between astrocytoma-like LGG with grade IV IDH-wild type tumors regarding possible worsening of treatment resistance along a proneural-to-mesenchymal axis. Using gene signaturebased inference we elucidated the impact of cellular composition of the tumors including immune cell bystanders such as macrophages.

(Continued on next page)
\end{abstract}

\footnotetext{
* Correspondence: binder@izbi.uni-leipzig.de

${ }^{\dagger} \mathrm{H}$. Binder and E. Willscher are contributed equally.

M. Weller and M. Loeffler are shared last authorship.

${ }^{1}$ Interdisciplinary Centre for Bioinformatics, Universität Leipzig, Härtelstr. 16-18,

04107 Leipzig, Germany

Full list of author information is available at the end of the article
}

(c) The Author(s). 2019 Open Access This article is distributed under the terms of the Creative Commons Attribution 4.0 International License (http://creativecommons.org/licenses/by/4.0/), which permits unrestricted use, distribution, and reproduction in any medium, provided you give appropriate credit to the original author(s) and the source, provide a link to the Creative Commons license, and indicate if changes were made. The Creative Commons Public Domain Dedication waiver (http://creativecommons.org/publicdomain/zero/1.0/) applies to the data made available in this article, unless otherwise stated. 
(Continued from previous page)

Conclusions: Genomic, epigenomic and transcriptomic factors act in concert but partly also in a decoupled fashion what underpins the need for integrative, multidimensional stratification of LGG by combining these data on gene and cellular levels to delineate mechanisms of gene (de-)regulation and to enable better patient stratification and individualization of treatment.

Keywords: Glioma, Molecular subtypes, DNA methylation, Epigenetics, Astrocytoma, Tumor microenvironment, Cellular composition, Prognosis

\section{Introduction}

Diffuse WHO grade II and III glioma (in short lower grade glioma, LGG) describe an almost fatal disease of young adults. These tumors share a more indolent course compared with high-grade IV gliomas (glioblastoma, GBM). Recent work has proposed a classification of glioma based mainly on two genetic markers, namely absence or presence of isocitrate dehydrogenase 1 and $2(I D H)$ mutation and of codeletion of chromosome arms $1 \mathrm{p}$ and $19 \mathrm{q}$ (codel), overriding histology [14, 37, 45, 56, 66]. IDH-mut codel tumors with mostly oligodendroglial histology are associated with the best prognosis; $I D H$-mut non-codel tumors with mostly astrocytic histology are associated with intermediate outcome; and $I D H$-wt, with mostly higher WHO grade (III or IV) tumors are associated with poor prognosis $[51,65]$. Besides genetic factors (DNA-)methylation has emerged an important regulator of gene transcription, and its role in tumorigenesis has become a topic of considerable interest $[11,33]$. A number of studies have reported alterations of DNA methylation in gliomas $[6,7,9,12,31,35,38,47,55]$.

$I D H$ mutations occur early in gliomagenesis in the vast majority of WHO grade II and III gliomas. They change the function of the IDH enzymes, causing them to produce 2-hydroxyglutarate (2HG), an oncometabolite that represses DNA demethylation [63] and, in consequence, leads to genome wide DNA-hypermethylation subsumed as glioma-CpG island methylator phenotype (G-CIMP) [47]. Whole genome methylation studies have revealed that G-CIMP gliomas split into subgroups differing in the Chr. 1p/19q codeletion status and the total level of methylation [8] where decreased methlylation associates with worse survival and increased risk for recurrence [12] and possibly reflects a global DNA demethylation shift of progressing G-CIMP-tumors. Both, genetic and epigenetic events can drive progression of gliomas leading to nearly identical phylo (epi-)genetic relations [40]. Moreover, recent studies reported continuous phenotypic drifts along a proneuronal-to-mesenchymal axis in $I D H$-wild type GBM associated with increasing therapy resistance that contradict a major role of genetic aberrations as drivers of essential tumor characteristics such as resistance [54] and that are linked to drifts in DNA methylation [32] and the cellular composition of the tumor microenvironment [62]. We ask if similar mechanisms can be identified also in IDH mutated LGG.

In general, deregulation of cell functions in cancer is encoded in both the genome and epigenome which underscores the importance of epigenetic analyses in parallel to the discovery of transcriptomics and genetics. Current analyses have not yet clarified the relationships between the methylome and transcriptome. In LGGs about $84 \%$ of all cases carry $I D H$-mutation with about $35 \%$ of them carrying an additional Chr. 1p/19q-codeletion, which enables studying phenotypic variability of the transcriptomes and methylomes especially of these genomic strata.

Our previous expression profiling of grade II and III primary glioma from a prospective German Glioma Network (GGN) cohort revealed rich heterogeneity of their transcriptomes which were only partially linked to the genomic features [65]. For this study transcriptomic and genetic data of the 137 lower grade glioma samples from the GGN cohort were complemented by new (DNA-) methylation data of 122 matched tumors of the same cohort which enables a combined analysis aimed to study DNA methylation as a shaping factor of glioma heterogeneity. Here we perform molecular subtyping which has emerged as an important concept to describe glioma heterogeneity and to better understand the biology of this devastating disease. We show that genomic, transcriptomic and methylation data provide partly overlapping but also distinct molecular subgroups, suggesting that different omics-views provide complementary and partly independent information about modes of gene-regulation $[26,57]$ with potentially different prognostic and therapeutic relevance. We aimed at characterizing the functional context of these different modes with special emphasis on the cellular composition of the tumors and their microenvironment and on possible impact for tumor development from lower grade to higher grade gliomas.

\section{Materials and methods}

\section{Patients, tumors and data}

The GGN study of WHO grade II or III gliomas (LGG) was described previously [65]. For this previous study, 
we had analyzed tumors of 137 patients by array-CGH, Affymetrix chip-based gene expression and candidate gene analyses (see [65] and Additional file 1: Figure S1). All tumors were subjected to central pathology review and classified according to the 2016 WHO classification of tumors of the central nervous system [37]. For the present study, molecular characterization was supplemented by array-based DNA methylation data (Illumina $450 \mathrm{~K}$ arrays) of 122 patient-matched tumors of the GGN cohort (Additional file 2: Table S1).

\section{Expression, CGH data and DNA methylation analyses}

Expression and array CGH data were processed as described in [65]. For genome-wide assessment of DNA methylation glioma samples were arrayed using the Illumina HumanMethylation450 BeadChip according to the manufacturer's instructions at the DKFZ as described previously [55]. A verification set of WHO grade II and III gliomas was taken from the TCGA repository including gene expression and DNA methylation data (Additional file 3: Table S3). Gene expression data were corrected for background noise, calibrated, quantile-normalized and transformed into log10-scale, as described in [65]. CpG IDs were mapped to the promoter region of each gene ranging from $2 \mathrm{~kb}$ upstream to $200 \mathrm{bp}$ downstream of the transcription start site using RefSeq mRNA annotation. DNA methylation beta-values of the respective $\mathrm{CpGs}$ were averaged to get one mean methylation beta-value for each gene promoter available. Genes located on Chr. X and Y were excluded from analyses. For an alternative analysis we also integrated CpG methylation over enhancer and gene body regions (see below).

\section{Bioinformatics analysis}

Gene expression and DNA methylation data were centralized and then analyzed after dimension reduction to metagenes using self-organizing map (SOM) machine learning [67]. As a result, each tumor tissue is characterized by the expression/methylation values of 2500/900 metagenes. Downstream analysis tasks including class discovery, visualization and knowledge mining using gene set analysis were performed with the R-package 'oposSOM' [36]. Unsupervised class discovery of expression and methylation subgroups was performed independently in metagene space by using maximum spanning graph-partitioning [65] followed by iterative maximization of the sample similarity score until convergence as described before [34]. For gene set profiling we applied the gene set Z-score (GSZ) metrics to estimate the mean differential expression of the set genes in each sample compared with their mean expression levels averaged over all samples in units of the respective standard deviation [58]. We considered gene sets related to biological processes (BP), of the gene ontology (GO) classification, and standard literature sets and literature sets curated by our group [68]. Immune cell composition of the tumor biopsies were estimated from the expression data using the program CIBERSORT [46].

\section{Results \\ WHO grade II/III gliomas split into eight expression and six methylation subtypes}

Single data type class discovery of gene expression data of 137 WHO grade II/III gliomas and DNA methylation data of patient-matched samples all collected in the German Glioma Network (GGN) provided eight expression subtypes designated as E1 - E8 (E-groups) and six methylation subtypes (M1 - M6, M-groups, Fig. 1a). The subtypes E1 and M1 nearly completely collect IDH-wild type tumors mostly with gains on Chr7 and losses of Chr10 representing genetic hallmarks of glioblastomas [5, 51, 65] (Fig. 1b, Additional file 1: Figure S2 and Additional file 1: Table S4 and Additional file 1: Table S5 for sample counts and enrichment analysis). The subtypes E2 - E6 and M2 - M5 nearly exclusively contain IDH mutated tumors predominantly without codeletions on Chr1p and Chr19q as genetic hallmarks of astrocytomas while the subtypes E6 and M5 strongly enrich samples with a codeletion on Chr1p and Chr19q as a genetic hallmark of oligodendrogliomas [37]. Gains on Chr7 that are not paralleled by losses on Chr10 are frequently found in E4 and M3 (Additional file 1: Figure S2). A chromosome map of gene expression reveals dose-response effects of all these chromosomal defects (Additional file 1: Figure S3A). We find a relatively high number of aberrations in E2 and a relatively small one in E7/M6 and E5/M4 (Fig. $1 \mathrm{~b}$ and Additional file 1: Figure S2). Interestingly, a bimodal differential methylation pattern between M1 - M3 (reduced methylation) and M4 - M6 (increased methylation) is detected for the olfactory subgenome [19] which collects genes encoding G-protein coupled receptors (GPCR's) especially on Chr11 (Additional file 1: Figure S3B). The E-groups do not show this clear separation into two entities although the amount of hypomethylated GPCR's increases progressively from E7 to E1 (Additional file 1: Figure S3B). A similar bimodal methylation patterns is found for gene clusters encoding keratin intermediate filament proteins on Chr12 and Chr17 in the M-groups (Additional file 1: Figure S3B). We compared the mean methylation levels of the promoter regions as used throughout this work with those of enhancer and gene body regions and found similar methylation patterns on average (Additional file 1: Figure S4), which suggests that aberrant methylation affects widespread genomic regions. 


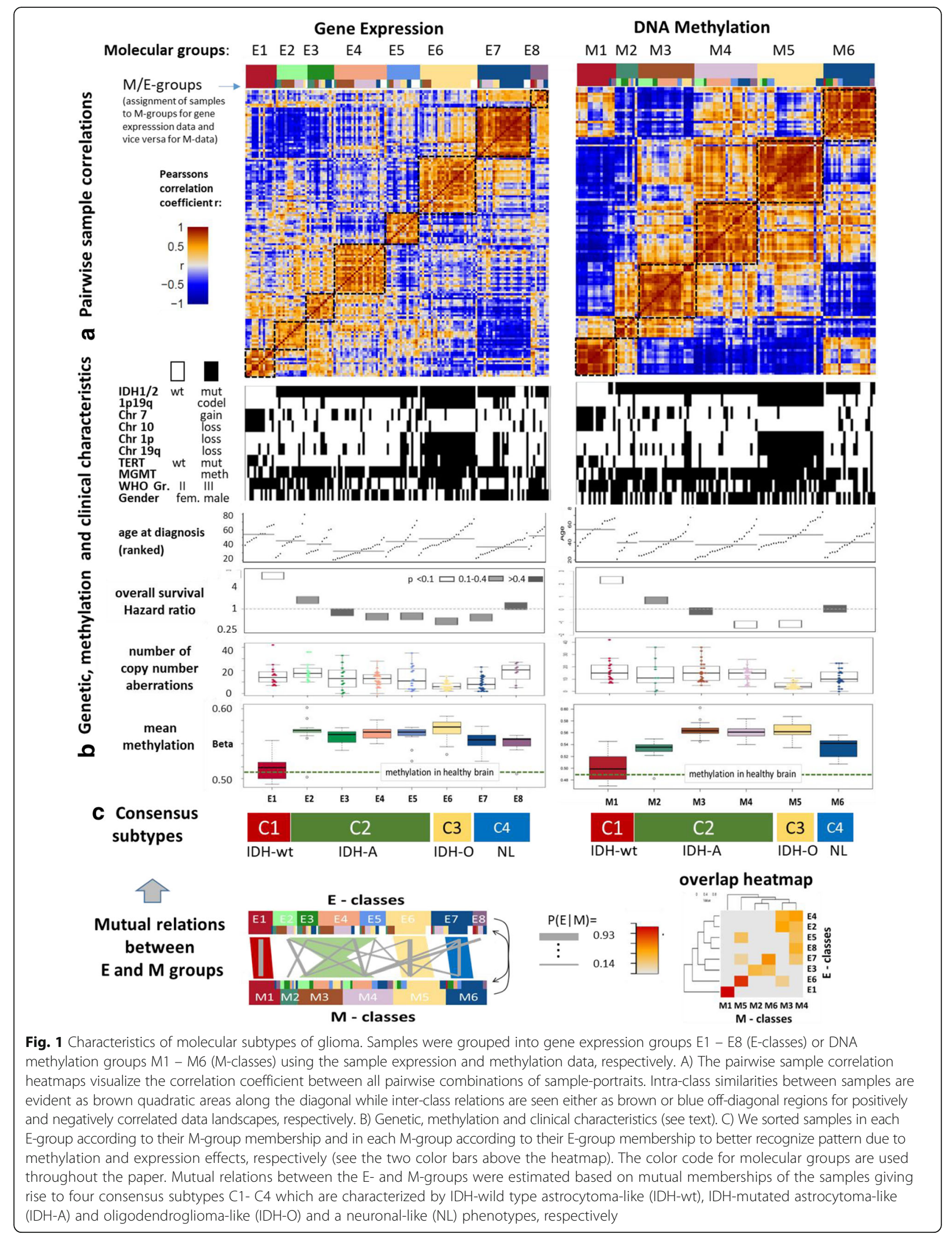


Consensus subtypes assign to astrocytoma-like, oligodendroglioma-like and neural phenotypes

Detailed analysis of the distribution of samples among the Eand $\mathrm{M}$-subtypes reveals large overlap of tumors and thus correspondence between E1 and M1, E6 and M5 and also between E7 and M6 (Fig. 1c) while E2 - E5 intermix with M2 - M4 with partial correspondence between E3 and M2. Based on these results we define the consensus classes $\mathrm{C} 1$ C4 where C1, C3 and C4 represent classes with almost one-to-one mutual correspondence between the expression and methylation subtypes. With a nearly exclusive content of $I D H$-wild type tumors in $\mathrm{C} 1$ (100\% in E1 and $87.5 \%$ in M1) and of $I D H$-mutated and Chr1p/19q codeleted tumors in C3 (92\% in E6 and 100\% in M5) these subtypes show clear genetic characteristics that assign them to expression and methylation phenotypes of $I D H$-wild type astrocytoma-like (IDH-wt) and to oligodendrogioma-like (IDH-O) resemblance, respectively [37]. In contrast, $\mathrm{C} 2$ is a more heterogeneous group regarding the correspondence between the Eand M-classes. It collects predominantly $I D H$ mutated tumors (more than 97\% in C2) almost always without Chr1p/ 19q codeletions (85\% for E-groups and 90\% for M-groups) and without alterations on Chr7 and Chr10 (95\%) (see also Additional file 1: Table S4) which assigns $\mathrm{C} 2$ to gliomas of $I D H$-mutant astrocytoma-like resemblance (IDH-A) [37]. Nevertheless, a minority of about $15 \%$ of all $I D H$-mutant and Chr.1p/19q codeleted oligodendrogliomas are in C2 (12.5\% in the E-groups and $17 \%$ in the M-groups) mostly because of a decreased methylation level of the GPCR subsumed in the olfactory subgenome that contrasts them compared with the majority of $67 \%$ oligodendrogliomas in C3/IDH-O (60\% in E6 and 75\% in M5) and also in C4 (15\% / 8\%, Additional file 1: Figure S3B). The consensus subtype C4 collecting E7, E8 and $\mathrm{M} 6$ constitutes mixtures of tumors with genetic characteristics present in all remaining subtypes. We assign specimens with reduced tumor cell content to $\mathrm{C} 4$ based on the observations that the mean number of copy number aberrations on Chr7 and Chr10 in E1 and on Chr1p and Chr19q in E6 is reduced for samples in E7, respectively (Additional file 1: Figure S5). Additionally, C4 shows a healthy brain functional context, e.g. related to synaptic transmission (see below). Hence, the expression and methylation landscapes of the glioma subtypes are shaped in first instance by the underlying key genetic defects in agreement with a recent classification of LGGs [56]. However, we also found a large degree of inter-tumoral heterogeneity of expression and methylation phenotypes that considerably modulates this genetic picture as illustrated by means of sample-similarity nets based either on the gene expression or on the methylation data (Additional file 1: Figure S7). This uncertainty obviously results, among other factors (such as tumor purity and composition), from the multidimensional nature of the transcriptomes and methylomes, e.g. from the combination of different G-CIMP- and GPCR-methylation patterns, from the lack of a clear-cut one-to-one relation between many of these phenotype-dimensions and the underlying genotypes.

\section{The subtypes differ in overall promoter methylation, WHO grade and prognosis}

Next, we compared the mean absolute promoter methylation level averaged over all genes and samples of each subtype (Fig. 1). It is low in $\mathrm{C} 1$ (IDH-wt) and high in C3 (IDH-O) and also C2 (IDH-A), as expected, because these predominantly $\mathrm{IDH}$-mutated tumors in $\mathrm{C} 2$ and $\mathrm{C} 3$ form the CpG hypermethylation phenotype (G-CIMP) [47]. The degree of hypermethylation in M2 is reduced compared with the other $I D H$ mutated tumors in C2 and $\mathrm{C} 3$ while promoter methylation is on intermediate level in $\mathrm{C} 4$ collecting a mixture of $I D H$-mutated (64\%) and $I D H$-wt $(36 \%)$ tumors. Interestingly, the mean methylation level of the subtypes inversely relates to their overall survival hazard ratio (Additional file 1: Figure S6). Worst prognosis of $I D H$ wild type LGG compared with $I D H$ mutated and Chr1p/19q codeleted (best prognosis) and non-codeleted ones (intermediate prognosis) was reported previously [65]. Interestingly, we find a similar, however more subtle inverse trend between methylation and HR in the E- and M-groups collected in $\mathrm{C} 2$ (IDH-A) that associates with the accumulation of WHO grade II astrocytic tumors in E4 (58\%) and E5 (60\%, decreased HR and increased methylation, Additional file 1: Table S4) while grade III tumors accumulate in E3 (100\%) and E2 (71\%, increased HR and decreased methylation). Enrichment of higher tumor grade III is also found in M2 (78\%) and M3 (70\%). It is associated with worse prognosis and decreased methylation (Additional file 1: Figure S6). Taken together, our data suggest associations between decaying methylation, increasing WHO grade and HR in $I D H$ mutated astrocytoma-like tumors (IDH-A).

\section{Verification using TCGA data and comparison with previous signatures of gliomas}

The E- and M-subtypes found here were confirmed (except E5) in more than 270 LGGs taken from The Cancer Genome Atlas (TCGA) using a guided SOM-extension method that combines the GGN- and TCGA-data and enables their joint analysis [34] (Additional file 1: Figure S8, Figure S9). Moreover, we selected a series of GBM and lower-grade glioma (LGG) signature gene sets of previous classification schemes and compared them with the subtypes identified herein (Fig. 2 and Additional file 1: Table S6). We found correspondence between our subtypes E1 and partly E3 and signatures of the classical (CL) and mesenchymal (ME) expression subtypes of grade IV gliomas [60], of the pre-glioblastoma (PG) subtype of LGG [20] and of hypermethylated genes of the 
G-CIMP-phenotype [47]. Signature genes of proneural (PN) GBM [60] and of early-progenitor-like (EPL) LGG [20] show similarities with C2 (IDH-A), with subtle differences between E2, E3 and E4, while neuronal GBM $(\mathrm{NL})$ and healthy brain signatures match to $\mathrm{C} 4$ and partly C3 (IDH-O). Interestingly, E3 collects $\mathrm{IDH}$-mutated glioma with an inflammatory, mesenchymal-like expression signature. The expression level of most of these signatures sharply change between the E-groups which indicates correspondence between our current classification and those previously described. The analysis of gene sets derived from methylation studies provides analogous results where, e.g., hypermethylation signatures in LGG [9] and the IDH subtype of GBM [55] largely agree with the G-CIMP-profile [47] that shows hypermethylation in M2 - M5 (Fig. 2). Oligodendroglial glioma reveal a modified G-CIMP-profile (G-CIMP-O) with enhanced methylation in M5 that closely resembles the RTKII profile [55]. CpG-level marker sets confirm the G-CIMP and G-CIMP-O profiles [49] (Additional file 1: Figure S10). Interestingly, methylation signatures of fetal and adult healthy brain [27] indicated strong similarity with the GPCR-signature meaning that the respective genes markedly lose methylation in gliomas, especially in M1 - M3. Overall, methylation signatures from previous studies including those of grade IV GBM [55] indicate similar underlying expression and methylation patterns. Accordingly, the consensus subtype $\mathrm{C} 1$ (IDH-wt) possesses pre-GBM (PG) characteristics, C2 (IDH-A) and C3 (IDH-O) are proneural-like (PN)

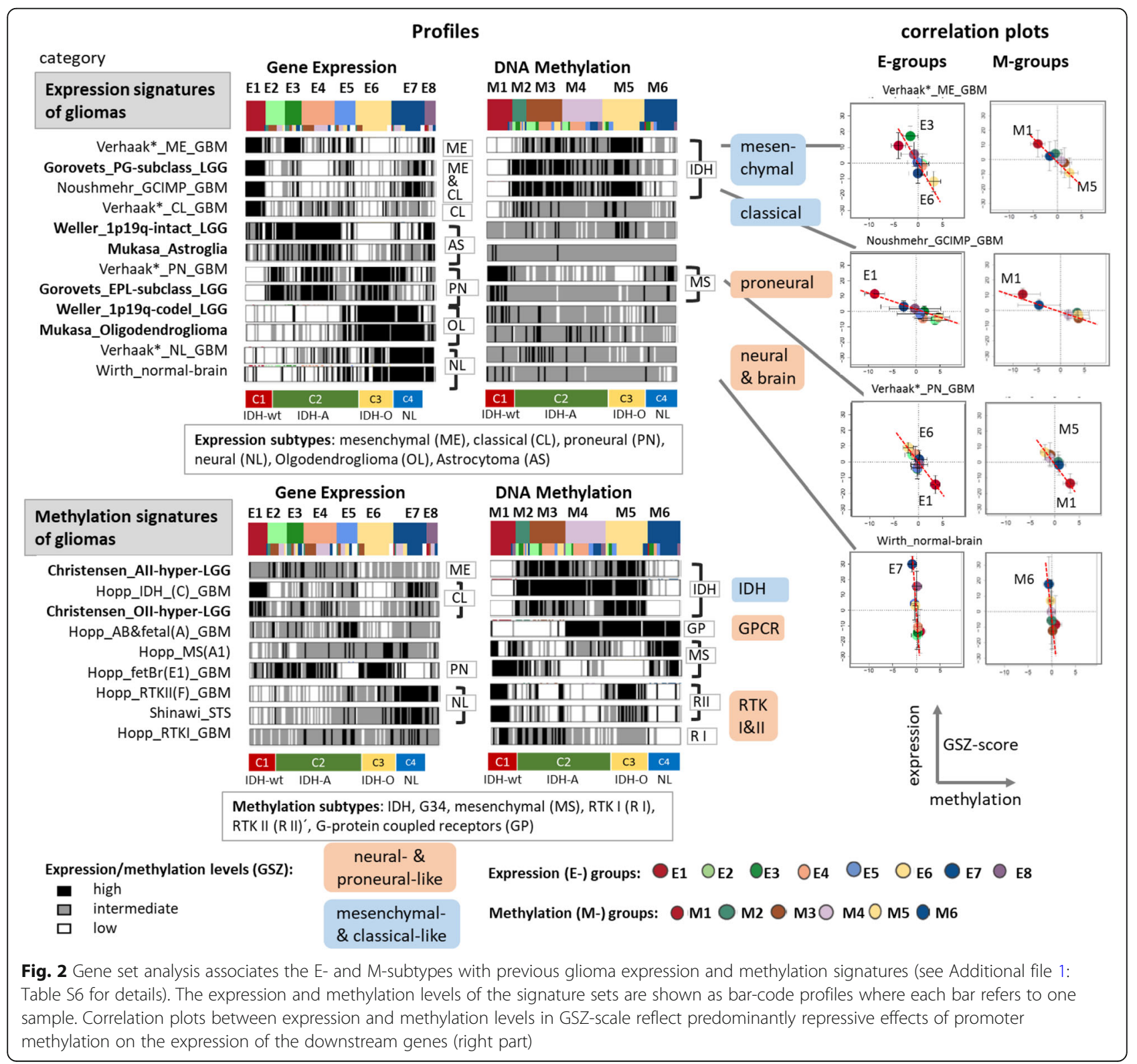


tumors (with E3 showing more mesenchymal-like characteristics) and C4 represents a neural-like (NL) subtype with mixed genetic characteristics of gliomas and expression properties partly resembling those of healthy brain in agreement with [62]. We also compared our subtypes with the epigenetic classes of Ceccarelli et al. [8] making use of CpG-level methylation and epigenetically regulated gene signatures (Additional file 1: Figure S11) and of an extended GGN data set (Additional file 1: Figure S13). Accordingly, M2 tumors reveal resemblance with the GCIMP-low and M3 - M4 tumors with the GCIMP-high classes of Ceccarelli et al. while C1 tumors can be assigned to CL-like (8 cases) and ME-like (6 cases) glioma based on their expression characteristics and to a $40 / 60 \%$ composition of RTKII and MS tumors using their methylation signatures. Most interestingly, the E3 tumors reveal characteristics of pilocytic astrocytomas (PA) which was detected by comparison with the expression patterns of 16 PA samples collected in the GGN. PA-resemblance was established for IDH-wt gliomas by Ceccarelli and colleagues but not for IDH-mut LGG. In summary, almost all E- and M- subtypes could be verified in an independent dataset and by previous glioma signatures where our approach stratifies IDH-mut astrocytomas (C2) in a novel way into three methylation and four expression subtypes which only partly match each to another.

\section{Functional context and epigenetic signatures}

Next we analyzed the functional context of the E- and M-subtypes of our data set (Fig. 3 and Additional file 1: Figures S14- S16). Gene signatures reflecting highly proliferating cells and high levels of oxphos metabolism are strongly expressed in E1 and E6 but weakly expressed in E7 which instead shows activated cell functions of healthy brain such as synaptic transmission. Inflammatory responses and a signature of epithelial-mesenchymal transition (EMT) were high in E3 and to a lesser degree observed in E1 but almost deactivated in C3 (IDH-O). Profiling of a series of metabolic gene sets confirms high oxphos and mitochondrial transcriptional activity in C3 paralleled by decreased glycolysis (Additional file 1: Figure S15) while C2 (IDH-A) is characterized by gained methylation and decreased expression of genes related to fatty acid metabolism, oxphos and mitochondrial functions. Interestingly, E2 seems metabolically deactivated throughout all processes considered while $\mathrm{C} 1$ (IDH-wt) shows the opposite trend.

Gene signatures of the ageing brain suggest parallels with inflammatory signatures upregulated in E3/E4. The methylation profiles of all these signatures show mostly anti-correlated patterns compared with the expression profiles (see the right 'methylation' part of Fig. 3a and Additional file 1: Figure S14). It indicates that promoter methylation predominantly represses transcription of the respective downstream gene. Gene sets estimating the activity of G-protein coupled receptors (GPCR), and of keratinization both show binary methylation patterns with low levels in M1 - M3 and higher levels in M4 M6 and correlated expression with the inflammatory signature and anti-correlated expression with the signature of synaptic transmission. Signatures related to epidermal cell differentiation and keratinization are prone to hypo-methylation also in other cancers [71]. They are found to tune the balance between stemness and somatic functions [28], to promote EMT-like processes [50] and also can serve as prognostic markers in epithelial cancers [30]. Testis-specific genes are overexpressed in E5. This phenomenon is observed also in other cancers [25] where so-called cancer testis (CT) genes often encode antigens that are thought to be immunogenic in gliomas and particularly in cancer stem cells $[16,18,73]$.

Interestingly, also signatures with impact for epigenetic mechanisms of gene regulation reflect pronounced subtype-specific differences (Fig. 3a, part below). Particularly, H3K4me3 marked genes in active promoters of neural progenitor cells (NPC) [42] and transcription factors (TF) associated with high expression levels in a wide collection of cells [24] show low methylation in M5 contrasted by high methylation in M1 and partly in M2 and M3 and thus similar trends as observed for the signatures related to highly proliferating cells and MYC targets discussed above. This seems plausible for M5 because highly proliferative cells require promoters activated by demethylation while activation of proliferation genes in M1 requires another mechanism. In contrast, hypermethylation in $\mathrm{C} 3$ (IDH-O), and to a less degree in $\mathrm{C} 1$ (IDH-wt) and C2 (IDH-A), is observed for targets of the polycomb repressive complex 2 (PRC2) in de-differentiated tumor cells [3], for related compounds such as SUZ12 and EED targets and for bivalently H3K4me3 and H3K27me3 marked genes in poised promoter states that are enriched in tumor suppressors [41]. Their suppression via hypermethylation promotes cancer development in gliomas and in other cancer entities [27]. The respective expression and methylation profiles closely resemble those of healthy brain and synaptic transmission thus suggesting their suppression by epigenetics in gliomas. It is known that $P R C 2$ is required for neuron specification during differentiation and for suppression of a transcriptional program that is detrimental to adult neuron function and which in case of $P R C 2$ deficiency leads to neurodegeneration via de-repression of bivalent $P R C 2$ target genes $[61,70]$. An analysis of genes in a set of defined chromatin states [15] determined in healthy fetal and adult brain tissues representing different states of brain development [52] further supports the view that suppressor-mechanisms in cellular programs are 


\section{a. Gene set analysis: Functional and epigenetic signatures}

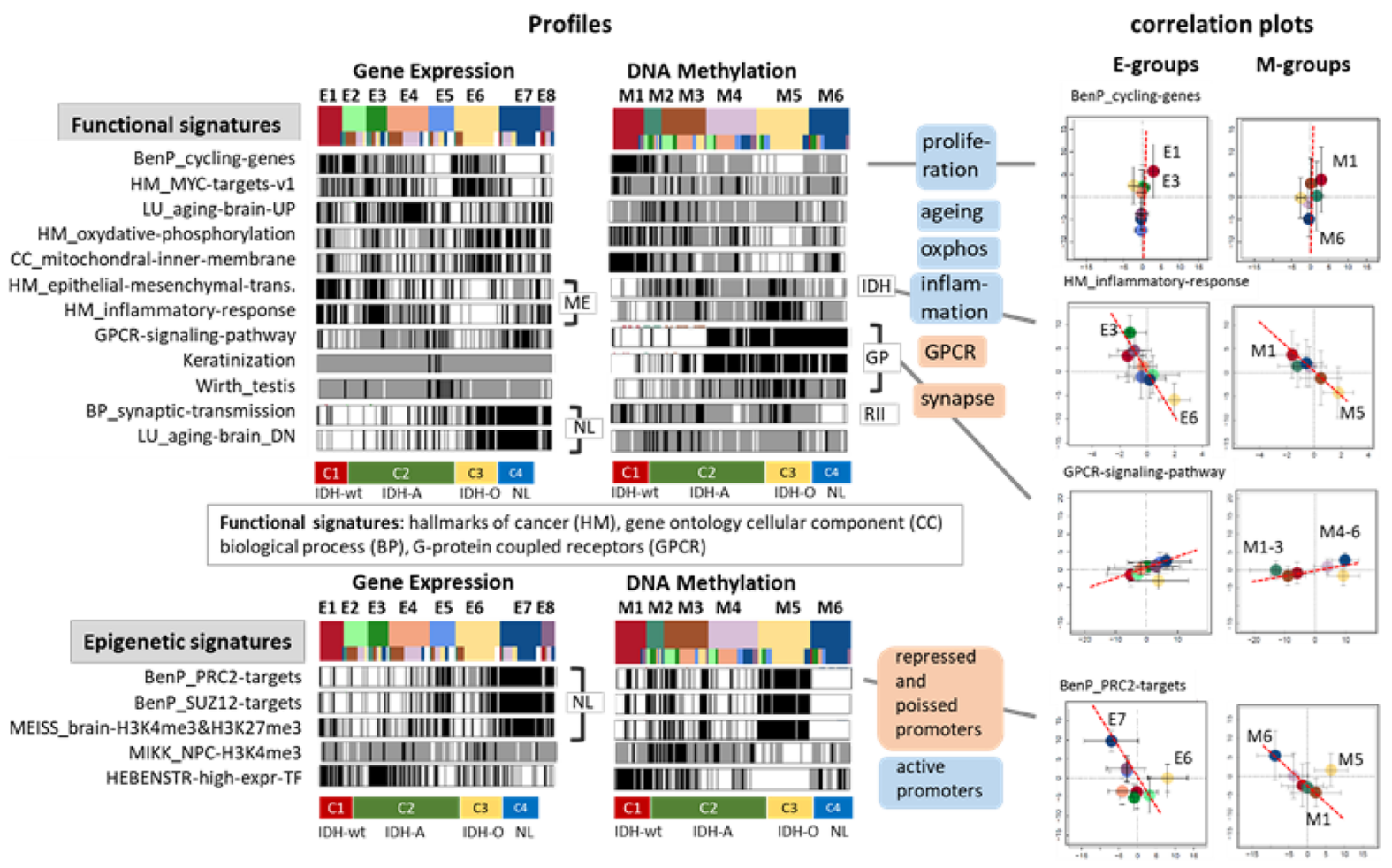

\section{b. Biological functions and glioma characteristics}
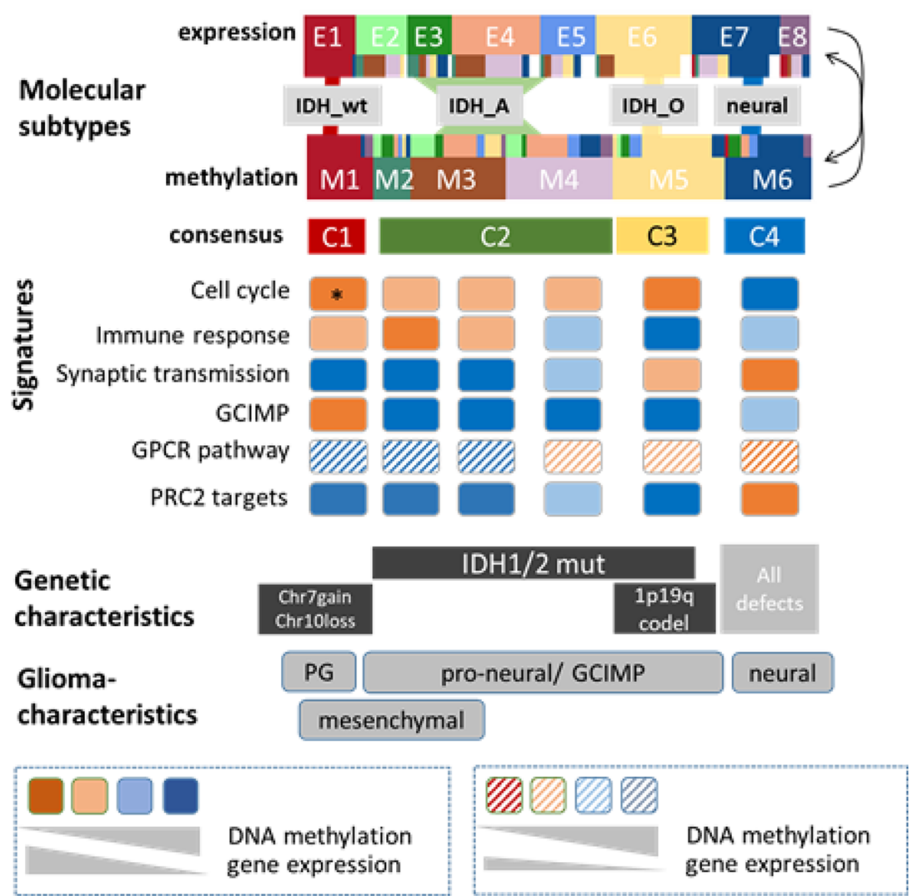

Fig. 3 Gene set analysis of functional and epigenetic signatures: a Bar-code profiles of expression and methylation levels of functional and epigenetic signatures and the correlation plots of subtype averaged values (see legend of Fig. 2). b Schematic overview about the basic functional, genetic and glioma characteristics extracted from the gene-signature analysis 
related to brain development and that genes in repressed states with impact for brain differentiation become hypermethylated in G-CIMP-subtypes and especially in C3 (Additional file 1: Figure S17).

Detailed functional analysis reveals anti-concerted alterations of expression and methylation, which associate with transcriptional activation of cell cycle related biological processes and the decay of neuronal processes such as synaptic transmission especially in $\mathrm{C} 1$ and $\mathrm{C} 3$ and also with changes of inflammatory characteristics in C2 (Additional file 1: Figure S18). Overall we identified three combined expression-methylation patterns (Additional file 1: Figure S19), namely (i) activating modes were related to proliferation and show increased expression which however associates either with increased (C1/ IDH-wt) or decreased (C3/IDH-A) methylation reflecting different driving mechanisms; (ii) deactivating modes which combine decreased expression and increased methylation in all subtypes associated with functions such as synaptic transmission; and (iii) functions related to immune response also showing anti-correlated changes between expression and methylation but an activating effect in $\mathrm{C} 1$ and especially E3 and deactivating effect in C3. Hence, degeneration of healthy brain functions in all subtypes, activated proliferation in C3 (IDH-O) and partly inflammation in E3 seem to be affected by anti-correlated DNA-promoter-methylation changes. In summary, the subtypes were characterized by combined alterations of the methylation and expression levels of genes from cellular programs such as proliferation, energy metabolism, immune response and synaptic transmission which associate with repressed and poised chromatin states in healthy brain and their subtype-specific remodeling in gliomas (Fig. 3b).

\section{Reference to single cell signatures disentangles glioma cell types}

Gliomas are composed of neoplastic and non-neoplastic cells, each of which potentially contribute to cancer formation, progression and response to treatment $[22,62]$. Bulk expression and methylation profiles as analyzed in this work average the diverse cell signatures within each tumor, thereby potentially masking critical differences and providing limited insight into cancer cell programs and the effect of the tumor microenvironment (TME). To disentangle this heterogeneity on cellular and TME-levels, we evaluated the expression and methylation degree of a collection of gene signatures taken from recent single cell RNAseq experiments on gliomas [59] in our data (Fig. 4a). We find that $\mathrm{C} 2$ gliomas were characterized by relatively high expression levels of benign astrocytes (astro-program, especially in E4), malignant astrocyte-like cells (IDH-A signature) and of microglia/macrophages (especially in E3) which all confirm the astrocyte-like phenotype of $\mathrm{C} 2$. On the other hand, these signatures are all low in C3 tumors which instead show activated expression of oligodendrocyte-like cells (oligo-program and $\mathrm{IDH}$-O signatures), of stemness and of neuronal signature genes where the latter ones are also high in C4 (neural subtype). The expression characteristics associate with almost mirror symmetrical methylation profiles showing either G-CIMP- or anti-G-CIMP characteristics, thus again suggesting regulatory effects of gene promoter methylation on downstream gene expression. A more detailed analysis indicates anti-correlated expression and methylation patterns of the malignant $I D H-\mathrm{A}$ and $I D H-\mathrm{O}$ dimensions suggesting that neoplastic transformations in $I D H-O$ and $I D H$-A cells are driven by de-methylation of the respective signature genes while cell cycle and microglia/ macrophage signatures increase and neuronal, healthy astro- and oligo-program signatures decline with increasing grade (Additional file 1: Figure S20). Overall, C3 tumors share closer similarities with healthy brain functions than C2-gliomas. C2-tumors instead show enhanced expression of macrophages/microglia signatures where microglia are crucial immune cells of the central nervous system and serve as tissue-resident macrophages of the brain [53]. On the other hand, both, C2 (IDH-A) and C3 (IDH-O) tumors are more proliferative compared with neuronal ones (C4). A higher amount of microglia/macrophage cells in astrocytoma and an increasing amount of proliferating cells is known to be a hallmark of higher grade gliomas [22]. In summary, the single cell characteristics reflect the variability of the composition of the tumors regarding healthy and benign astrocyte- and oligodendrocyte-like cells, microglia/macrophage and proliferative stem cell-like constituents in the bulk specimens studied.

\section{mRNA inferred immune cell components}

To further characterize the TME we employed CIBERSORT [46], a computational cell-type deconvolution method that decomposes the glioma-associated stroma into its immunological cellular components using cell-type related expression signatures (Fig. 4b and Additional file 1: Figure S21). We find that M2-macrophages are highly abundant in the astrocytic groups E1 - E4 with highest levels in E3 and E2 opposed by a reduction in monocytes. M2-macrophages play a pro-tumoral and anti-inflammatory role in brain cancer; they promote tissue remodeling and tumor growth [13, 53], particularly in glioma [43], and associate with resistance to radiotherapy in mesenchymal glioblastoma [62]. In contrast, anti-tumoral and pro-inflammatory M1-macrophages are almost absent in all grade LGG subtypes except for the pre-GBM subtype E1. Beyond a dual M1/M2 polarization status, a continuum between M1 and 
a. Single-cell signatures (Venteicher et al. 2017)
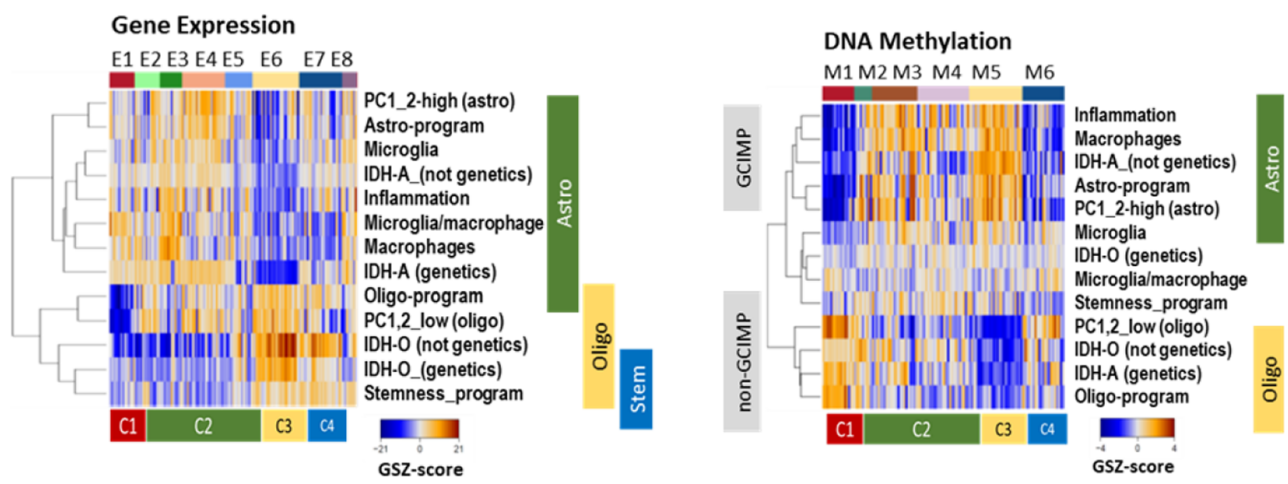

b. Immune cell decomposition (Cibersort)
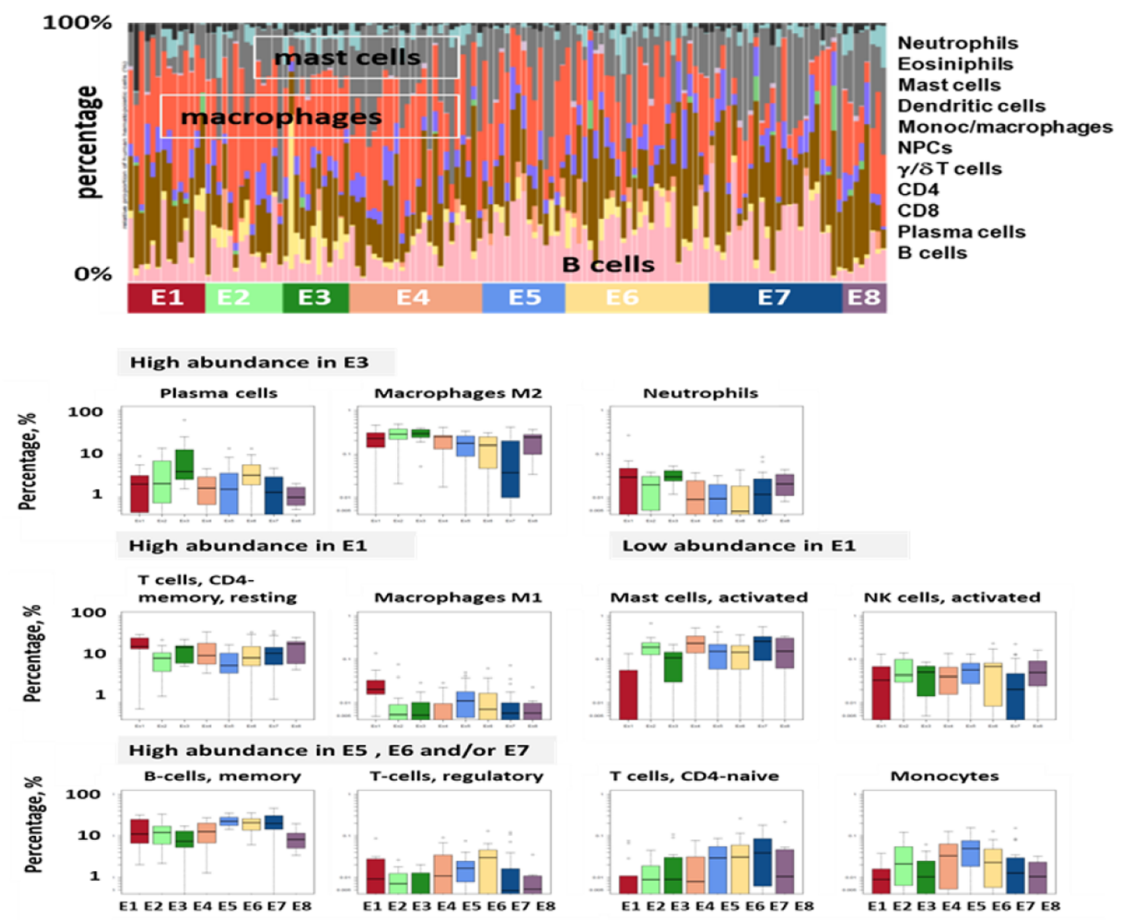

C. Drug and radiation resistance signature (Segerman et al. 2016)
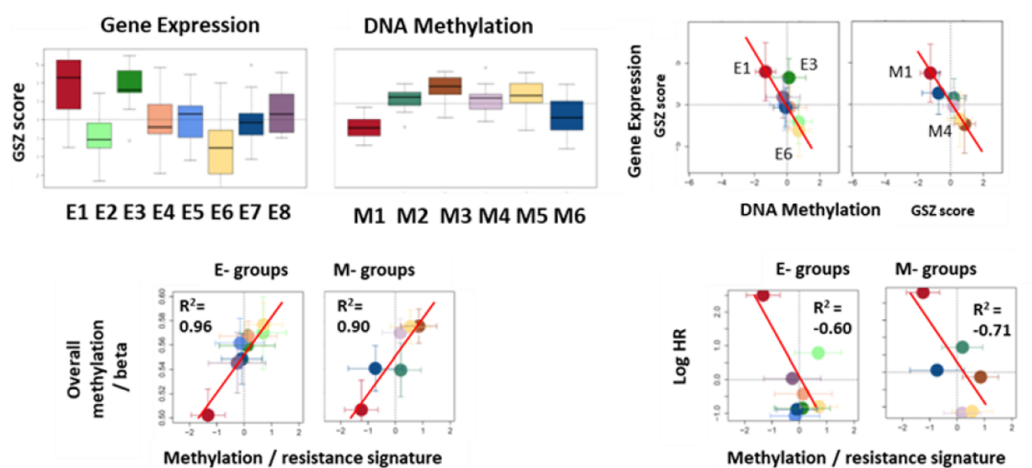

Fig. 4 (See legend on next page.) 
(See figure on previous page.)

Fig. 4 Cell type, micro-environmental immune cell and treatment-resistance characteristics. a Heatmaps of expression and methylation levels of single-cell signatures taken from [59] reveal subtype-specific activation of astrocyte-, oligodendrocyte- and stem cell-like characteristics. b Digital immune cell-type decomposition of glioma transcriptomes using CIBERSORT [46] (see Fig. S21 for the full set of cells considered) on sample (above) and mean subtype levels for selected leukocyte cells across the expression subtypes. c The boxplots of expression and methylation levels of a transcriptomic drug and radiation resistance signature containing 50 genes [54] suggest largest resistance effects in E3 and E1. Expression and methylation levels of the subgroups anti-correlate (right part)

M2 polarization seems to exists in glioma [17] including IDH-mut tumors [59] which provides a possible interpretation of the increasing M2 percentage in C2 from E5 to E3. It has been hypothesized that the most aggressive and invasive cells in GBM are neoplastic macrophages arising in fusion hybrids between neoplastic stem cells and macrophages/microglia [29]. The high M2-macrophage abundance in astrocytic gliomas is paralleled by relatively large percentages of neutrophils while increased abundance of M1-macrophages in E1 is accompanied by CD4-resting memory T-cells. The amount of tumor infiltrating CD4+ leukocytes in glioblastoma correlates with tumor progression and presumably relates to tumor angiogenesis [23, 44]. We also found that activated mast cells are relatively abundant in virtually all groups (especially in E4, E5 and E7) except E1. Mast cells were shown to become recruited and 'educated' by glioma cells in a glioma grade-dependent manner to reduce stemness, decrease proliferation and migration to induce differentiation of glioma cells [1]. This mechanism seems to apply to early tumor stages of $I D H$-mutated astrocytoma-like gliomas (C2). Interestingly, regulatory T-cells (Tregs) show increased percentage in the oligodendroglioma-like subtype C3, the subtype with lowest immune and inflammatory characteristics, which is in correspondence with the immunosuppressive role of Tregs in glioma [48]. We also make use of immune cell gene signatures taken from [4] to compare their expression and methylation levels (Additional file 1: Figure S21). We find that most of them show high expression especially in E3 and E1 reflecting their accumulation in higher grade astrocytoma. These expression profiles are mostly paralleled by G-CIMP and especially G-CIMP-O methylation profiles which suggest deactivation of immune cell activities in C3 by DNA methylation. Interestingly, the methylation profile of T-cells resembles that of GPCR, which suggests a cell specific relation between DNA methylation and gene expression. Hence, the changes of methylation observed originate from both glioma and immune cells, which suggests coupled epigenetic mechanisms during tumor development. Note that DNA methylation in glioma bulk samples was found to be predictive for immune cell infiltration [32]. In summary, digital immune cell deconvolution of the transcriptome reveals that M2-macrophages were enriched in higher grade astrocytomas (E1- E3) while activated mast cells are more abundant in the neuronal subtype (C4), in lower grade astrocytomas (E4- E5) and in oligodendrogliomas (E3) together with immunosuppressive Tregs. Hence, the TME is characterized by marked variations of the immune cell composition that overlays with methylation changes of their genomes which suggests an epigenetically-mediated interplay between development of tumor cells and immune cells in the TME.

\section{Treatment resistance and senescence signatures associate with methylation}

Next, we studied a 50-gene multi-therapy resistance signature, which reflects a continuum of cell phenotypes with increasing resistance against chemo- and radiotherapy paralleled by a proneural-to-mesenchymal shift of their transcriptomes [54]. In our data, we find a profile of this signature showing highest expression in E3 (C2) and $\mathrm{C} 1$ and lowest in C3 (Fig. 4c), thus suggesting a gradient of treatment resistance from oligodendroglioma-like to astrocytoma-like tumors with inflammatory characteristics of the TME. The resistance signature resembles the profiles of inflammatory and EMT functional signatures (Fig. 3a) and that of the mesenchymal GBM-subtype (Fig. 2a) in our data. Tumors of the latter type indeed showed enhanced treatment resistance $[32,54,62]$. The methylation profile of the resistance signature reflects G-CIMP characteristics and anti-correlates with the respective expression levels, which suggest a methylation-driven repression mechanism. Interestingly, the methylation profile of the resistance signature strongly correlates with the total methylation level of the tumors $\left(R^{2}>0.9\right)$, which suggests that treatment resistance associates with overall methylation of the tumors. Our data support the view that the methylation profile of the signature anti-correlates with the HR-profile $\left(R^{2}<-0.7\right.$, compare also with Fig. 1) showing that worsening of prognosis of astrocytomalike $I D H$-mutated tumors (C2/IDH-A) associates with de-methylation of the tumors. A GCIMP-low methylation profile, mesenchymal-like expression characteristics and genomic instability was recently found in recurrent gliomas [12] in analogy with the characteristics of M2/E3-tumors reported here (Additional file 1: Figure S2, Figure S12).

A recent model of glioma progression suggests that increased senescence bypass mechanisms proceed in parallel with tumor development and the formation of a pro-inflammatory microenvironment at later phases [2]. We, therefore, studied a signature of genes that contribute to senescence bypass mechanisms by promoterhypermethylation during aging and tumorigenesis and which associate with cancer risk [69]. These genes become 
increasingly deactivated in the tumors of the E-groups from E7 to E1, i.e. along the neuronal- proneural to mesenchymal axis (Additional file 1: Figure S23). Their senescence profile resembles those of the PRC2-targets, RTKII-characteristics, ageing and healthy brain signatures, while the methylation profiles of the two latter signatures differ from the former ones regarding methylation of Chr1p/19q-codeleted tumors in C3. Particularly, these tumors show increased methylation of senescence genes accompanied by demethylation and transcriptional upregulation of genes involved in oxphos-metabolism (Fig. 3 and Additional file 1: Figure S15) and/or deactivated inflammatory response. It is assumed that $\mathrm{Chr} 1 \mathrm{p} / 19 \mathrm{q}$ codeleted gliomas (C3) bypass senescence by other mechanisms than Chr1p/19q-non-codeleted tumors [2]. Overall, the LGG-subtypes group along a therapy-resistance signature suggesting that resistance and recurrence are mediated by epigenetics and an inflammatory TME along the proneural mesenchymal-like axis also in LGG accompanied by graded loss of methylation and increased $\mathrm{CNV}$ and IDH-wt resemblance. Moreover, astrocytoma-like tumors in C2 seem to develop along this axis as indicated by progressive activation of senescence bypass mechanisms.

\section{Discussion}

\section{Heterogeneity of WHO grade II and III gliomas}

Our multi-platform transcriptome-methylome-genome study revealed a large molecular heterogeneity of adult diffuse gliomas of WHO grades II and III: we identified eight expression and six methylation subtypes and characterized them in terms of genetic aberrations, functional context, cellular composition, tumor microenvironment and their possible impact on treatment resistance and prognosis as illustrated in the summary scheme in Fig. 5. The expression and methylation patterns of the glioma subtypes are shaped by the underlying key genetic defects in agreement with recent classifications of LGG [8, 45, 56]. Overall, we identified three consensus subtypes C1-C3 that were assigned as $I D H$-wt and $I D H$-mut astrocytoma-like and oligodendroglioma-like phenotypes according to their dominating genetic status in terms of the $I D H$ mutation and Chr. 1p/19q codeletion. These genetic aberrations are assumed to act as early events of tumorigenesis [64] (see left part of Fig. 5a). A fourth, neuronal subtype (C4) collects specimen with reduced tumor cell content and served as reference partly resembling characteristics of healthy brain. However, our subtypes reflect also a large variability of expression and methylation phenotypes that do not match the genetic hallmarks in a one-to-one fashion. For example, $25-40 \%$ of all $I D H$-mut and $1 \mathrm{p} / 19 \mathrm{q}$-codel tumors were not assigned to the oligodendroglioma-like subtype (C3) but rather resemble the astrocytoma-like $(\mathrm{C} 2)$ or neuronal $(\mathrm{C} 4)$ types by a series of features. This heterogeneity results, among other possible factors, from the multidimensional nature of the transcriptomes and methylomes of the tumors. Each of their expression and methylation landscapes can be interpreted as a superposition of different expression and methylation patterns, which associate with specific cellular and micro-environmental states, and which obviously lack a clear-cut relation with respect to the underlying genotypes. The astrocytoma like gliomas constitute the largest fraction of nearly $60 \%$ of all LGG studied. They revealed the largest diversity and were divided into four expression (E2-E5) and three methylation (M2-M4) subtypes, which only partly match each other, thus reflecting partly decoupled expression and methylation patterns due to different possible interaction mechanisms [26, 57]. Particularly, decoupling between transcription and methylation can be rationalized in terms of independent regulation mechanisms of transcription by epigenetic and transcription factor (TF)-networks which are governed by bistable epigenetic switches [57]. Applying this model to cell differentiation data we recently identified situations where variant transcription of genes is accompanied by invariant epigenetic promoter states or vice versa. Interestingly, the former situation of TF-dominated regulation seems to apply to elementary cell functions related to stress response, cell cycle regulation and cell metabolism and requires mostly high expression levels of the genes beyond the sensitivity range of the switches. Combined regulation is found for developmental processes where genes become activated or deactivated by epigenetics, usually via histone methylation changes associated with DNA-hypo- or hypermethylation near their promoters. Changes of methylation with only minor effect on transcription was found for GPCRs also upon cell differentiation. Overall we find striking agreement between gene functions in these three regimes between cell developmental data [57], WHO grade IV GBM [26] and the LGG studied here. Interestingly, methylation seems to-activate enhancers in TF-networks while it de-activates enhancers for developmental processes [72] or, in other words, enhancer and promoter methylation seem to act in an antagonistic fashion for both types of processes. A more simplistic interpretation of partly decoupled expression and methylation assumes rarely or non-overlapping sets of 'passenger' genes regulated by TFs and/or epigenetic 'drivers' such as the IDH-mutation [26].

On a cellular level, our results support a multicomponent approach underpinned by single-cell transcriptome characteristics [59] that indicates variable composition of the tumors regarding healthy astrocyteand oligodendrocyte-like cells, microglia/macrophage and proliferative stem cell-like constituents as illustrated in the right part of Fig. 5a. The TME of the subtypes is characterized by marked variations of the immune cell composition that overlays with methylation changes of 


\section{a. Phenotypic heterogeneity of LGG}
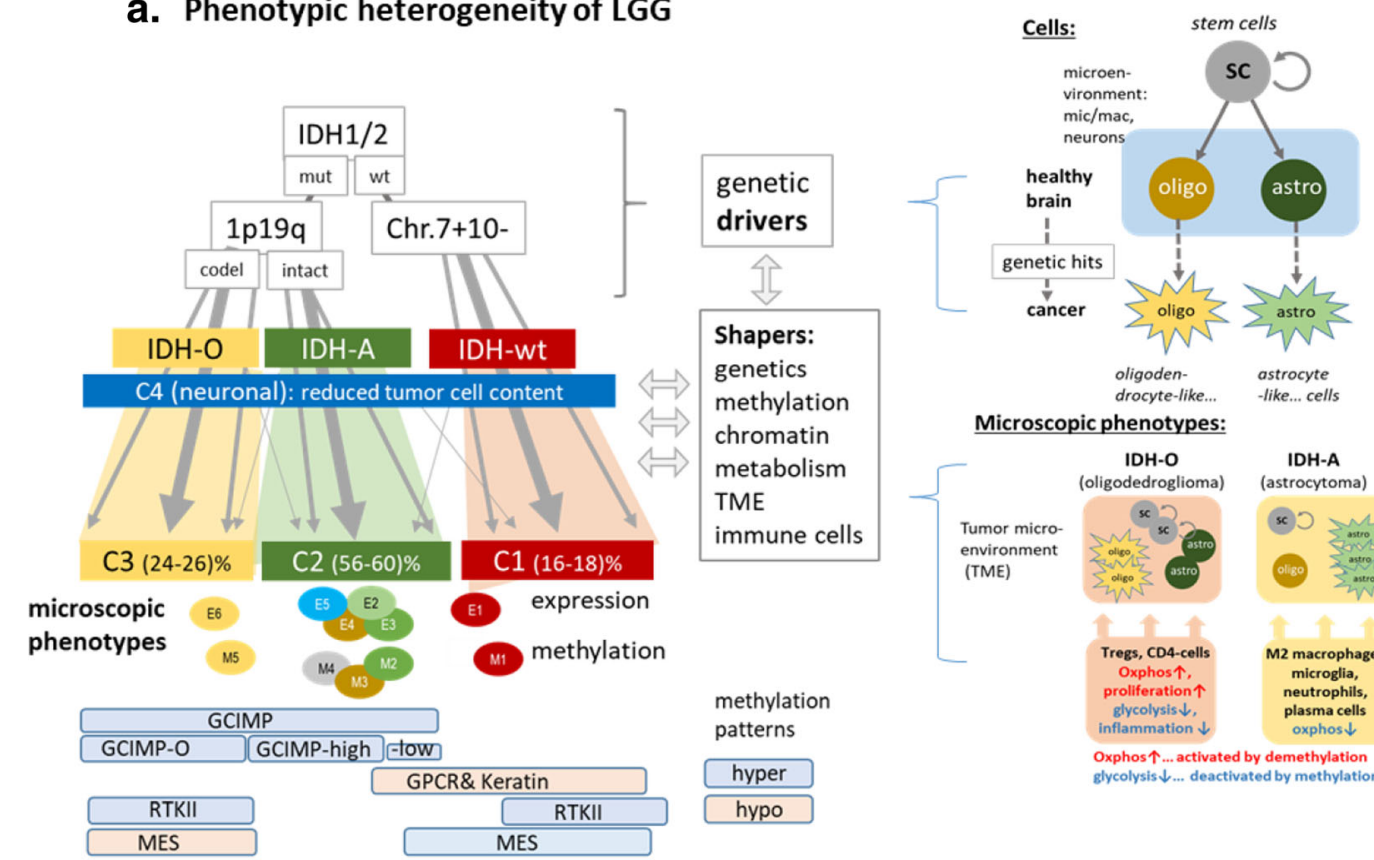

Microscopic phenotypes:

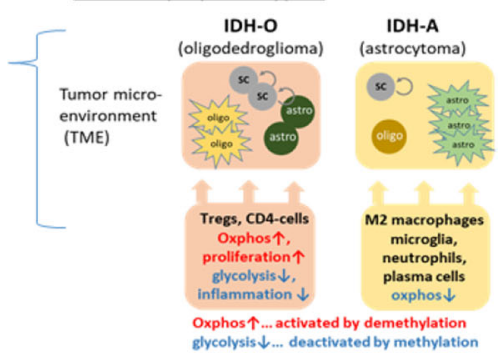

b. Phenotypic trees and functional profiles
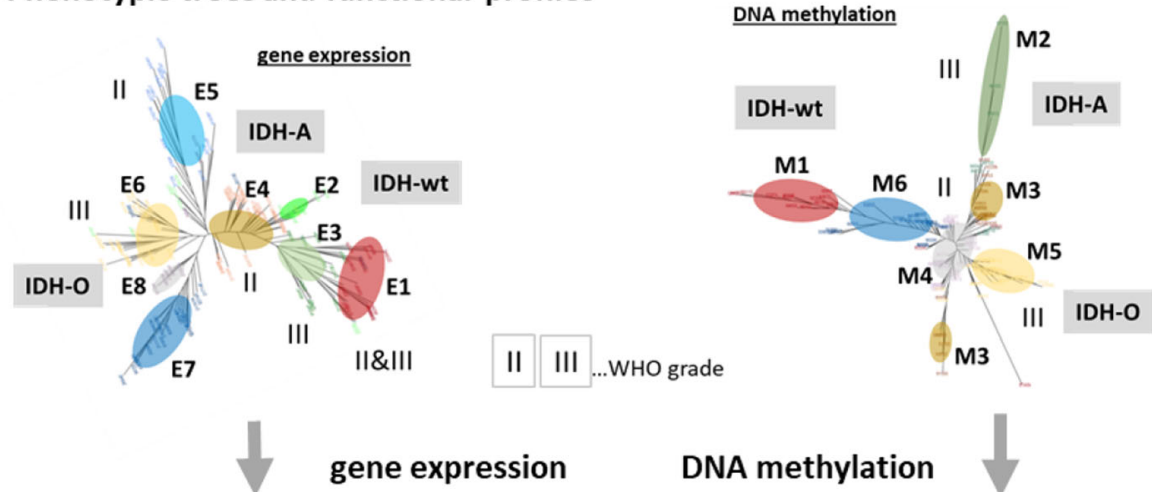

DNA methylation
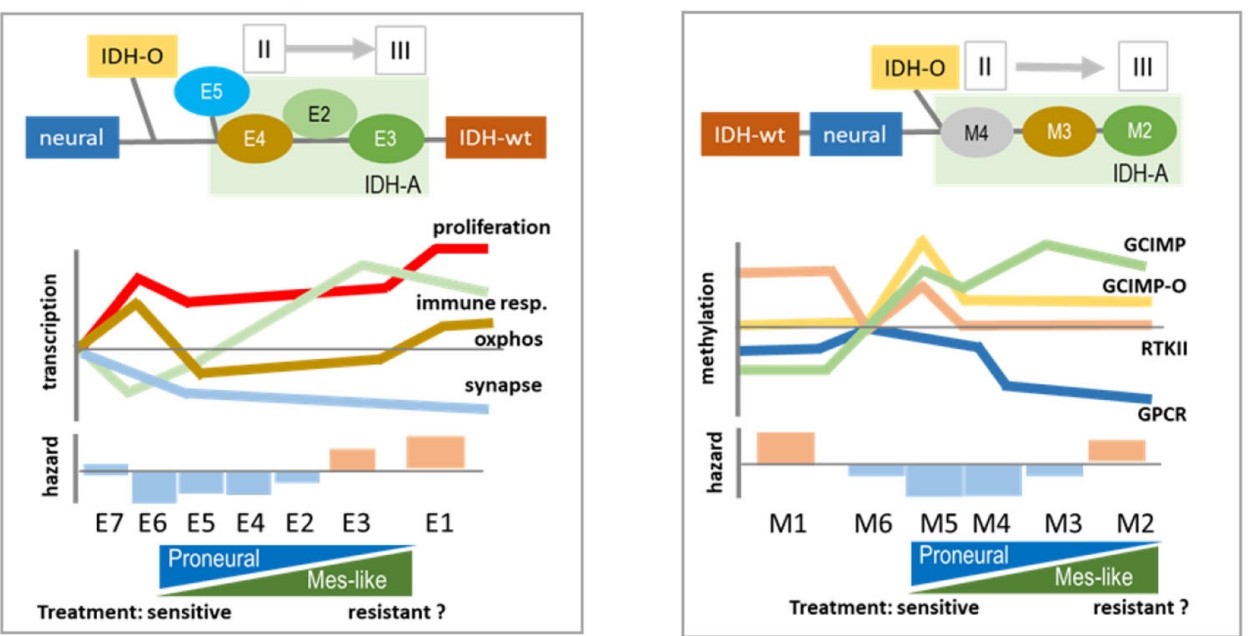

Fig. 5 (See legend on next page.) 
(See figure on previous page.)

Fig. 5 Schematic summary: a The major glioma subtypes arise after specific genetic hits. The tumor phenotypes are then shaped by the tumor microenvironment (TME), its cell composition, epigenetics and additional genetic defects. Different methylation patterns develop in a subtype specific fashion upon tumor progression (left part). On a cellular level, astrocyte-like and oligodendrocyte-like gliomas are both primarily composed of proliferating stem cells, oligodendrocytes and astrocytes, however in different amounts, which associates with different immune cell compositions in the TME and metabolic expression signatures, which partly are affected by methylation effects. b Phenotypic trees provide similarity relations between the expression and methylation subtypes (top), which were simplified as one-dimensional sequences of subtypes and associated with selected transcriptional programs, methylation patterns and prognosis (bottom)

their genomes. It suggests an epigenetically-mediated interplay between tumor cells and immune cells in the TME.

We found footprints of previously published expression and methylation gene signatures extracted from studies on WHO grade II, III and IV gliomas in the tumors studied here indicating a considerable overlap of molecular mechanisms between LGG and GBM [8] in agreement with previous studies which underlined relevance of GBM molecular signatures for LGG [21]. These results support the view that the molecular heterogeneity of gliomas decomposes into a set of gene-regulatory modes that were activated in different combinations and to a varying extent in the individual subtypes and in tumors of different grades. In addition to the G-CIMP and G-CIMP-O signatures that typically occur in $\mathrm{IDH}$-mut gliomas we also found methylation characteristics occurring in $I D H$-wt GBM such as the RTK II and mesenchymal methylation signatures reflecting concerted methylation changes of respective groups of genes in IDH-mut LGG as well (Fig. 5a, part below). Moreover, we found concerted methylation patterns of the olfactory subgenome collecting GPCR genes and of cancer-related keratin intermediate filament genes, respectively. These signatures overlap and combine in different ways giving rise to diverse methylation and expression patterns that partly shape the glioma phenotypes.

\section{Phenotypic relatedness suggests developmental paths of gliomas}

For a more detailed view on the relatedness between the subtypes we performed similarity tree analysis of the molecular tumor landscapes (Fig. 5b). The expression and methylation 'phenotypic' trees obtained differ mainly in the position of the $I D H$-wt $(\mathrm{C} 1)$ subtype. Its expression characteristics show rather similarities with the $\mathrm{C} 2$ tumors because of common inflammatory and astrocytic signatures while its methylation profiles rather resemble that of neuronal (C4) tumors owing to the common lack of the G-CIMP patterns (see the schematic profiles in the lower part of Fig. $5 \mathrm{~b}$ ). On the other hand, both trees reflect similar mutual relations between the neuronal, oligodendroglioma-like (C3) and astrocytoma-like (C2) tumors where the former two types share similarities mainly regarding (low) inflammatory, (high) neuronal expression and (high) GPCR-methylation levels. Degeneration of apparent healthy brain functions in all subtypes, activated proliferation in C3 and partly inflammation in E3 seem to be driven by anti-correlated DNA-promoter methylation changes.

Interestingly, the astrocytoma-like subtypes in $\mathrm{C} 2$ sort in the order E4-E2-E3 and M4-M3-M2, respectively, which associates with increasing WHO grade of the tumors, their age at first diagnosis, their hazard ratio, the decrease of the global methylation levels and of neuronal expression characteristics and increased senescence bypass characteristics. We hypothesize that these trends reflect aspects of the progression of astrocytoma like gliomas from earlier to later phases in the natural course of the disease $[2,8]$. Interestingly, these trends also suggest increasing therapy resistance along the proneuralto-mesenchymal axis after comparison with resistance and inflammatory signatures derived from GBM [54]. Search for glioma subtypes and/or molecular characteristics most suitable for immunotherapies is a challenge [43]. The inflammatory subtype E3 with maximum M2-macrophage polarization could be of interest for therapies targeting glioma associated macrophages [10].

Importantly, decreasing total methylation decomposes into reduced methylation of the GPCR- and keratinmethylation patterns on one hand, and the G-CIMP pattern, which shows the opposite trend in M4-M3, on the other hand (Fig. 5b, part below). The relative reduction of G-CIMP in M2 is compatible with the observation that while IDH-mut associated G-CIMP initiates gliomagenesis it seems not required for later clonal expansions [39]. Interestingly, the RTKII-signature originally obtained from WHO grade IV IDH-wild type gliomas shows parallels with the senescence bypass signature in $I D H$-mutated LGG, and particularly reflects differences between Chr1p/ 19q-codeleted and -non-codeleted tumors. Both, loss and gain of methylation take place in parallel in different regions of the genome of tumor cells and/or in different cellular constituents of the TME. The subtype E5 manifests characteristics of early stages of astrocytoma-like tumors such as high levels of the GPCR- and keratin-methylation patterns and low levels of the G-CIMP-methylation in addition to the expression of cancer testis genes. E5 collects both, Chr1p/19q-non-codeleted (mainly grade II) and, to a less amount, Chr1p/19q-codel tumors, which suggests that mechanisms affecting DNA methylation act partly independent of the Chr1p/19q-codel status. 


\section{Aberrant methylation shapes glioma phenotypes}

Gliomas are consistently characterized by the loss of neuronal expression signature, especially in $I D H$-wt and $I D H$-mut astrocytoma-like, and to a less degree, also in oligodendroglioma-like tumors, paralleled by decreasing expression and hyper-methylation of $P R C 2$-targets with possible consequences for senescence bypass mechanisms. The latter properties are hallmarks of CIMP-like subtypes observed in colon cancer and lymphoma and in grade IV glioma [27, 38]. Previous studies have proposed a role for $P R C 2$ genes in protecting neurons against degeneration by repressing aberrant transcriptional programs [61]. Stratification of repressed chromatin states in fetal and adult brain revealed an antagonistic methylation pattern between oligodendroglioma-like (C3) and $I D H$-wt gliomas and an intermediate pattern in astrocytoma-like (C2) tumors which suggests deregulation of developmental cellular programs in $I D H$-wt; and of programs of differentiated tissue in $\mathrm{C} 3$ and partly also in $\mathrm{C} 2$. The former effect associates with the activation of inflammation and mesenchymal characteristics while the second one seems to activate proliferation and oxphos metabolism. Epigenetic activation of otherwise suppressed cellular programs seems to be essential for glioma development and diversification into subtypes.

Our methylation analysis uses integral methylation beta-values of upstream regions of each gene which are assumed to reflect mean promoter methylation levels. Similar methylation patterns were found in extended upstream regions, in the gene body and also for CpG-related signatures, which all together suggests that DNA-methylation affects widespread genomic regions in a similar fashion. On the other hand, this integral methylation analysis eventually overlooks local and CpG-specific methylation effects with possible impact for transcriptional regulation. In this context, our integral method should be judged as one limitation of this study. We expect that alternative methods will further improve our understanding of the role of DNA-methylation, e.g., to better resolve the regulatory element landscapes and transcription factor networks [72] in gliomas. Also the possible impact of methylation of the olfactory subgenome on cell function and glioma development remains partly unclear and requires future work.

\section{Conclusions}

Our study demonstrates the importance of molecular subtyping of LGG as a concept to better understand the biology of this disease. We hereby follow a holistic approach which is guided by previous findings that diffuse gliomas can be further divided into epigenomic subtypes that differ in their biology with impact for treatment and prognosis beyond the WHO classification and histopathological grade. IDH mutated astrocytoma-like LGGs constitute the most heterogeneous sub-entity, which stratifies into distinct transcriptomic and methylation subtypes with possible impact for clinics, e.g. for identification of treatment resistant or sensitive tumor strata. Analogies between astrocytoma-like LGG with grade IV $I D H$-wt tumors regarding varying treatment resistance suggest similar disease mechanisms; however further studies are required for verification. Hereby epigenetics, and particularly, DNA methylation is a shaping and driving factor of glioma heterogeneity and progression. Genomic, epigenetic and transcriptomic factors act in concert but partly also in a decoupled fashion what underpins the need for integrative, multidimensional subtyping of LGG by combining these data on gene and cellular levels in order to delineate mechanisms of gene (de-)regulation and to enable better patient stratification for individualization of treatment.

\section{Additional files}

Additional file 1: Tables S3-S6. Contains all supplementary figures and supplementary tables. (PDF $2870 \mathrm{~kb}$ )

Additional file 2: Table S1. List of GGN-patients whose DNA methylation data were included into the study. (XLSX $12 \mathrm{~kb}$ )

Additional file 3: Table S2. List of TCGA-samples used for verification analyses. (XLSX $18 \mathrm{~kb}$ )

\section{Abbreviations}

2HG: 2-hydroxyglutarate; BP: Biological process; C1 - C4: Consensus classes of LGG with mutual correspondence between expression and methylation; CL: Classical subtype of grade IV gliomas according to (Verhaak, et al., 2010); CN: Copy number; E1 - E8: Expression subtypes of LGG; EMT:

Epithelial-mesenchymal transition; EPL: Early-progenitor-like subtype; G-CIMP: Glioma CpG-Island hyperMethylation Phenotype; G-CIMP-O: G-CIMP with specific hypermethylation of IDH-O; G-CIMP-wt: Hypermethylation of IDH-wt, particularly of the RTK II type; GEO: Gene expression omnibus database; GGN: German Glioma Network; GPCR: G-protein coupled receptor; GSZ: Gene set Z-score; HM: Hallmarks of Cancer; HR: Hazard ratio; IDH: Isocitrate dehydrogenase; IDH-A: Malignant astrocytoma-like; IDH-mut non-codel: IDH mutant with euploid 1p/19q; IDH-mut-codel: IDH mutant additionally carrying codeletion of chromosome arm 1p and 19q; IDH-O: Malignant oligodendrocytoma-like; IDH-wt : IDH wild-type; LGG: Lower grade gliomas (WHO grade II and III); M1 - M6: Methylation subtypes; ME: Mesenchymal subtype of grade IV gliomas according to (Verhaak, et al., 2010); MGMT: O-6-methylguanine-DNA methyltransferase; MP: Methylation pattern; MS: Mesenchymal subtype (Sturm et al. (reanalyzed by Hopp et al.)); NL: Neural subtype of grade IV gliomas according to (Verhaak, et al., 2010); NPC: Neural progenitor cells; OS HR : Overall survival hazard ratio; Ox: Oxidative phosphorylation; PG: Pre-glioblastoma subtype of LGG (Gorovets, et al., 2012); PN: Proneural subtype of grade IV gliomas according to (Verhaak, et al., 2010); PRC2: Polycomb repressive complex 2; RTK: Receptor tyrosine kinase; RTKI: GBM methylation class (Sturm et al., 2012); RTKII: GBM methylation class (Sturm et al., 2012); SOM: Self-organizing map; TF: Transcription factors; TME: Tumor microenvironment

\section{Funding}

This work is supported by the Federal Ministry of Education and Research (BMBF), project grant SysGlio and the DKTK joint funding project "Next generation molecular diagnostics of malignant gliomas" and the German Glioma Network (GGN).

Availability of data and materials

Expression data are available in the gene expression omnibus (GEO) database under accession number GSE61374 [73]. DNA methylation data reported in this study for the first time are deposited in the gene expression 
omnibus (GEO) database under accession number GSE129477 The complete data are available from 'The Leipzig Health Atlas' repository (https://www. health-atlas.de/lha/7vkp0crkfe-5).

\section{Authors' contributions}

Coordinator of GGN: MW. Conceived and performed analysis, wrote manuscript: HB, EW. Performed special bioinformatics analyses and developed methods: LH, HLW. Methylation experiments and primary analysis: DTJ, MP. Performed analyses, contributed to the study and the manuscript: all authors. All authors read and approved the final manuscript.

\section{Ethics approval and consent to participate}

All patients gave written informed consent for participation in the GGN and its translational research projects.

\section{Consent for publication}

Not applicable.

\section{Competing interests}

MW has received research grants from Abbvie, Acceleron, Actelion, Bayer, Merck, Sharp \& Dohme (MSD), Merck (EMD), Novocure, OGD2, Piqur, Roche and Tragara, and honoraria for lectures or advisory board participation or consulting from Abbvie, BMS, Celgene, Celldex, Merck, Sharp \& Dohme (MSD), Merck (EMD), Novocure, Orbus, Pfizer, Progenics, Roche, Teva and Tocagen. US has received honoraria for lectures or advisory board participation from medac, GSK, mundipharma, Novartis, Novocure, Roche. DK has received research grants from Novocure, Northwest biotherapeutics, Kyowa, and honoraria for lectures or advisory board participation or consulting from Baxter and Kyowa. WW received study drug for clinical research from Apogenix, Roche and Pfizer. JCT has received research grants BrainLab and honoraria for lectures from BrainLab, Siemens, Merck, Roche and medac.

All other authors declare that they have no competing interests.

\section{Publisher's Note}

Springer Nature remains neutral with regard to jurisdictional claims in published maps and institutional affiliations.

\section{Author details}

'Interdisciplinary Centre for Bioinformatics, Universität Leipzig, Härtelstr. 16-18, 04107 Leipzig, Germany. ${ }^{2}$ Hopp Children's Cancer Center Heidelberg (KiTZ), Im Neuenheimer Feld 430, 69120 Heidelberg, Germany. ${ }^{3}$ Pediatric Glioma Research Group, German Cancer Research Center (DKFZ), Im Neuenheimer Feld 280, 69120 Heidelberg, Germany. ${ }^{4}$ Division of Pediatric Neurooncology, German Cancer Consortium (DKTK), German Cancer Research Center (DKFZ), Im Neuenheimer Feld 280, 69120 Heidelberg, Germany. ${ }^{5}$ Department of Pediatric Oncology, Hematology and Immunology, Heidelberg University Hospital, Im Neuenheimer Feld 430, 69120 Heidelberg, Germany. ${ }^{6}$ Institute for Medical Informatics, Statistics and Epidemiology, University of Leipzig, Härtelstraße 1618, 04107 Leipzig, Germany. ${ }^{7}$ Department of Neurology, University Hospital and University Zurich, Frauenklinikstrasse 26, 8091 Zurich, Switzerland. ${ }^{8}$ Clinic for Neurosurgery, Tübingen University Hospital, Hoppe-Seyler-Straße 3, 72076 Tübingen, Germany. ${ }^{9}$ Division of Clinical Neurooncology, Department of Neurology, University Hospital Bonn, Bonn, Germany. ${ }^{10}$ Institute of Neuropathologie, University Clinic Hamburg-Eppendorf, Martinistraße 52, 20246 Hamburg, Germany. "Department of Neurosurgery, University Clinic Hamburg-Eppendorf, Martinistraße 52, 20246 Hamburg, Germany.

${ }^{12}$ Department of Neurosurgery, Technical University Dresden, Fetscherstraße 74, 01307 Dresden, Germany. ${ }^{13}$ Department of Neurosurgery, Ludwig Maximilians University Munich and German Cancer Consortium (DKTK), partner site Munich, Marchioninistraße 15, D-81377 Munich, Germany. ${ }^{14}$ Department of Neurology, University Hospital Knappschaftskrankenhaus Bochum-Langendreer, In der Schornau 23-25, 44892 Bochum, Germany. ${ }^{15}$ Clinic for Neurosurgery, University Düsseldorf, Moorenstr. 5, 40225 Düsseldorf, Germany. ${ }^{16}$ Clinical Cooperation Unit Neurooncology, German Cancer Consortium (DKTK), German Cancer Research Center (DKFZ), Im Neuenheimer Feld 280, 69120 Heidelberg, Germany. ${ }^{17}$ Neurology Clinic and National Center for Tumor Diseases, University Hospital Heidelberg, Im Neuenheimer Feld 400, 69120 Heidelberg, Germany.

${ }^{18}$ Department of Human Genetics, Hannover Medical School, Carl-Neuberg-Str. 1, 30625 Hannover, Germany.
Received: 17 January 2019 Accepted: 18 March 2019 Published online: 25 April 2019

\section{References}

1. Attarha S, Roy A, Westermark B, Tchougounova E (2017) Mast cells modulate proliferation, migration and stemness of glioma cells through downregulation of GSK3 $\beta$ expression and inhibition of STAT3 activation. Cell Signal 37:81-92

2. Barthel FP, Wesseling P, Verhaak RGW (2018) Reconstructing the molecular life history of gliomas. Acta Neuropathol 135:649-670

3. Ben-Porath I, Thomson MW, Carey VJ, Ge R, Bell GW, Regev A, Weinberg RA (2008) An embryonic stem cell-like gene expression signature in poorly differentiated aggressive human tumors. Nat Genet 40:499-507

4. Bindea G, Mlecnik B, Tosolini M, Kirilovsky A, Waldner M, Obenauf AC (2013) Spatiotemporal dynamics of intratumoral immune cells reveal the immune landscape in human cancer. Immunity 39:782-795

5. Brat DJ, Aldape K, Colman H, Holland EC, Louis DN, Jenkins RB, Kleinschmidt-DeMasters BK, Perry A, Reifenberger G, Stupp R et al (2018) CIMPACT-NOW update 3: recommended diagnostic criteria for "diffuse astrocytic glioma, IDH-wildtype, with molecular features of glioblastoma, WHO grade IV". Acta Neuropathol 136:805-810

6. Brennan Cameron W, Verhaak Roel GW, McKenna A, Campos B, Noushmehr H, Salama Sofie R, Zheng S, Chakravarty D, Sanborn JZ, Berman Samuel $H$ et al (2013) The somatic genomic landscape of glioblastoma. Cell 155:462-477

7. Capper D, Jones DTW, Sill M, Hovestadt V, Schrimpf D, Sturm D, Koelsche C, Sahm F, Chavez L, Reuss DE et al (2018) DNA methylation-based classification of central nervous system tumours. Nature 555:469

8. Ceccarelli M, Barthel Floris P, Malta Tathiane M, Sabedot Thais S, Salama Sofie R, Murray Bradley A, Morozova O, Newton Y, Radenbaugh A, Pagnotta Stefano M et al (2016) Molecular profiling reveals biologically discrete subsets and pathways of progression in diffuse glioma. Cell 164:550-563

9. Christensen BC, Smith AA, Zheng S, Koestler DC, Houseman EA, Marsit CJ, Wiemels JL, Nelson HH, Karagas MR, Wrensch MR et al (2011) DNA methylation, Isocitrate dehydrogenase mutation, and survival in glioma. J Natl Cancer Inst 103:143-153

10. Cinzia Dello R, Lucia L, Lucio T, Pierluigi N, Grazia G, Colin KC (2017) Exploiting microglial functions for the treatment of glioblastoma. Curr Cancer Drug Targets 17:267-281

11. Das PM, Singal R (2004) DNA methylation and Cancer. J Clin Oncol 22:4632-4642

12. de Souza CF, Sabedot TS, Malta TM, Stetson L, Morozova O, Sokolov A, Laird PW, Wiznerowicz M, lavarone A, Snyder J et al (2018) A distinct DNA methylation shift in a subset of glioma CpG Island Methylator phenotypes during tumor recurrence. Cell Rep 23:637-651

13. Domingues P, González-Tablas M, Otero Á, Pascual D, Miranda D, Ruiz L, Sousa P, Ciudad J, Gonçalves JM, Lopes MC et al (2016) Tumor infiltrating immune cells in gliomas and meningiomas. Brain Behav Immun 53:1-15

14. Eckel-Passow JE, Lachance DH, Molinaro AM, Walsh KM, Decker PA, Sicotte H, Pekmezci M, Rice T, Kosel ML, Smirnov IV et al (2015) Glioma groups based on 1p/19q, IDH, and TERT promoter mutations in tumors. N Engl J Med 372:2499-2508

15. Ernst J, Kellis M (2010) Discovery and characterization of chromatin states for systematic annotation of the human genome. Nat Biotech 28:817-825

16. Freitas MRP, Malheiros SMF, Stávale JN, Biassi TP, Zamunér FT, de Souza Begnami MDF, Soares FA, Vettore AL (2013) Expression of Cancer/testis antigens is correlated with improved survival in glioblastoma. Oncotarget 4:636-646

17. Gabrusiewicz K, Rodriguez B, Wei J, Hashimoto Y, Healy LM, Maiti SN, Thomas G, Zhou S, Wang Q, Elakkad A et al (2016) Glioblastoma-infiltrated innate immune cells resemble M0 macrophage phenotype. JCI Insight 1: e85841

18. Ghafouri-Fard S, Modarressi M-H (2012) Expression of cancer-testis genes in brain tumors: implications for cancer immunotherapy. Immunotherapy 4:59-75

19. Glusman G, Yanai I, Rubin I, Lancet D (2001) The complete human olfactory subgenome. Genome Res 11:685-702

20. Gorovets D, Kannan K, Shen R, Kastenhuber ER, Islamdoust N, Campos C, Pentsova E, Heguy A, Jhanwar SC, Mellinghoff IK et al (2012) IDH mutation and neuroglial developmental features define clinically distinct subclasses of lower grade diffuse astrocytic glioma. Clin Cancer Res 18: 2490-2501 
21. Guan X, Vengoechea J, Zheng S, Sloan AE, Chen Y, Brat DJ, O'Neill BP, de Groot J, Yust-Katz S, Yung W-KA et al (2014) Molecular subtypes of glioblastoma are relevant to lower grade glioma. PLoS One 9:e91216

22. Hambardzumyan D, Gutmann DH, Kettenmann H (2016) The role of microglia and macrophages in glioma maintenance and progression. Nat Neurosci 19:20-27

23. Han S, Zhang C, Li Q, Dong J, Liu Y, Huang Y, Jiang T, Wu A (2014) Tumour-infiltrating CD4+ and CD8+ lymphocytes as predictors of clinical outcome in glioma. Br J Cancer 110:2560

24. Hebenstreit D, Fang M, Gu M, Charoensawan V, van Oudenaarden A, Teichmann SA (2011) RNA sequencing reveals two major classes of gene expression levels in metazoan cells. Mol Syst Biol 7:497

25. Hofmann O, Caballero OL, Stevenson BJ, Chen Y-T, Cohen T, Chua R, Maher CA, Panji S, Schaefer U, Kruger A et al (2008) Genome-wide analysis of cancer/testis gene expression. Proc Natl Acad Sci U S A 105:20422-20427

26. Hopp L, Löffler-Wirth H, Galle J, Binder H (2018) Combined SOM-portrayal of gene expression and DNA methylation landscapes disentangles modes of epigenetic regulation in glioblastoma. Epigenomics 10:745-764

27. Hopp L, Willscher E, Wirth-Loeffler H, Binder H (2015) Function shapes content: DNA-methylation marker genes and their impact for molecular mechanisms of glioma. J Cancer Res Updates 4:127-148

28. Huang K, Shen Y, Xue Z, Bibikova M, April C, Liu Z, Cheng L, Nagy A, Pellegrini M, Fan J-B, Fan G (2014) A panel of CpG methylation sites distinguishes human embryonic stem cells and induced pluripotent stem cells. Stem Cell Reports 2:36-43

29. Huysentruyt LC, Akgoc Z, Seyfried TN (2011) Hypothesis: are neoplastic macrophages/microglia present in glioblastoma multiforme? ASN neuro 3:e00064

30. Karantza V (2011) Keratins in health and cancer: more than mere epithelial cell markers. Oncogene 30:127-138

31. Kloosterhof NK, Bralten LBC, Dubbink HJ, French PJ, van den Bent MJ (2011) Isocitrate dehydrogenase-1 mutations: a fundamentally new understanding of diffuse glioma? The Lancet Oncology 12:83-91

32. Klughammer J, Kiesel B, Roetzer T, Fortelny N, Nemc A, Nenning K-H, Furtner J, Sheffield NC, Datlinger P, Peter N et al (2018) The DNA methylation landscape of glioblastoma disease progression shows extensive heterogeneity in time and space. Nat Med 24(10):1611-1624

33. Klutstein M, Nejman D, Greenfield R, Cedar H (2016) DNA methylation in Cancer and aging. Cancer Res 76:3446-3450

34. Kunz M, Löffler-Wirth H, Dannemann M, Willscher E, Doose G, Kelso J, Kottek T, Nickel B, Hopp L, Landsberg J et al (2018) RNA-seq analysis identifies different transcriptomic types and developmental trajectories of primary melanomas. Oncogene 37(47):6136-6151

35. Laffaire J, Everhard $S$, Idbaih $A$, Crinière $E$, Marie $Y$, de Reyniès $A$, Schiappa R, Mokhtari K, Hoang-Xuan K, Sanson M et al (2011) Methylation profiling identifies 2 groups of gliomas according to their tumorigenesis. Neuro-Oncology 13:84-98

36. Löffler-Wirth H, Kalcher M, Binder H (2015) oposSOM: R-package for high-dimensional portraying of genome-wide expression landscapes on bioconductor. Bioinformatics 31:3225-3227

37. Louis DN, Perry A, Reifenberger G, von Deimling A, Figarella-Branger D, Cavenee WK, Ohgaki H, Wiestler OD, Kleihues P, Ellison DW (2016) The 2016 World Health Organization classification of tumors of the central nervous system: a summary. Acta Neuropathol 131:803-820

38. Martinez R, Martin-Subero Il, Rohde V, Kirsch M, Alaminos M, Fernández AF, Ropero S, Schackert G, Esteller M (2009) A microarray-based DNA methylation study of glioblastoma multiforme. Epigenetics 4:255-264

39. Mazor T, Chesnelong C, Pankov A, Jalbert LE, Hong C, Hayes J, Smirnov IV, Marshall R, Souza CF, Shen Y et al (2017) Clonal expansion and epigenetic reprogramming following deletion or amplification of mutant $<$ em $>\mid \mathrm{DH} 1<$ /em $>$. Proc Natl Acad Sci 114:10743-10748

40. Mazor T, Pankov A, Johnson BE, Hong C, Hamilton EG, Bell RJA, Smirnov IV, Reis GF, Phillips JJ, Barnes MJ et al (2015) DNA methylation and somatic mutations converge on cell cycle and define similar evolutionary histories in brain tumors. Cancer Cell 28:307-317

41. Meissner A (2010) Epigenetic modifications in pluripotent and differentiated cells. Nat Biotech 28:1079-1088

42. Mikkelsen TS, Ku M, Jaffe DB, Issac B, Lieberman E, Giannoukos G, Alvarez P, Brockman W, Kim T-K, Koche RP et al (2007) Genome-wide maps of chromatin state in pluripotent and lineage-committed cells. Nature 448:553-560

43. Morisse MC, Jouannet S, Dominguez-Villar M, Sanson M, Idbaih A (2018) Interactions between tumor-associated macrophages and tumor cells in glioblastoma: unraveling promising targeted therapies. Expert Rev Neurother 18:729-737

44. Mu L, Yang C, Gao Q, Long Y, Ge H, DeLeon G, Jin L, Chang Y, Sayour EJ, Ji $J$ et al (2017) CD4+ and perivascular Foxp3+ T cells in glioma correlate with angiogenesis and tumor progression. Front Immunol 8:1451

45. Network TCGAR (2015) Comprehensive, integrative genomic analysis of diffuse lower-grade gliomas. N Engl J Med 372:2481-2498

46. Newman AM, Liu CL, Green MR, Gentles AJ, Feng W, Xu Y, Hoang CD, Diehn M, Alizadeh AA (2015) Robust enumeration of cell subsets from tissue expression profiles. Nat Methods 12:453

47. Noushmehr H, Weisenberger DJ, Diefes K, Phillips HS, Pujara K, Berman BP, Pan F, Pelloski CE, Sulman EP, Bhat KP et al (2010) Identification of a CpG Island Methylator phenotype that defines a distinct subgroup of glioma. Cancer Cell 17:510-522

48. Ooi YC, Tran P, Ung N, Thill K, Trang A, Fong BM, Nagasawa DT, Lim M, Yang I (2014) The role of regulatory T-cells in glioma immunology. Clin Neurol Neurosurg 119:125-132

49. Paul Y, Mondal B, Patil V, Somasundaram K (2017) DNA methylation signatures for 2016 WHO classification subtypes of diffuse gliomas. Clin Epigenetics 9:32-32

50. Polioudaki H, Agelaki S, Chiotaki R, Politaki E, Mavroudis D, Matikas A, Georgoulias V, Theodoropoulos PA (2015) Variable expression levels of keratin and vimentin reveal differential EMT status of circulating tumor cells and correlation with clinical characteristics and outcome of patients with metastatic breast cancer. BMC Cancer 15:399

51. Reifenberger $G$, Weber RG, Riehmer V, Kaulich $\mathrm{K}$, Willscher E, Wirth $\mathrm{H}$, Gietzelt J, Hentschel B, Westphal M, Simon M et al (2014) Molecular characterization of long-term survivors of glioblastoma using genome- and transcriptome-wide profiling. Int J Cancer 135:1822-1831

52. Roadmap Epigenomics Consortium, Kundaje A, Meuleman W, Ernst J, Bilenky M, Yen A, Heravi-Moussavi A, Kheradpour P, Zhang Z, Wang J et al (2015) Integrative analysis of 111 reference human epigenomes. Nature 518:317-330

53. Roesch S, Rapp C, Dettling S, Herold-Mende C (2018) When immune cells turn bad-tumor-associated microglia/macrophages in glioma. Int J Mol Sci 19:436

54. Segerman A, Niklasson M, Haglund C, Bergström T, Jarvius M, Xie Y, Westermark A, Sönmez D, Hermansson A, Kastemar M et al (2016) Clonal variation in drug and radiation response among glioma-initiating cells is linked to proneural-mesenchymal transition. Cell Rep 17:2994-3009

55. Sturm D, Witt H, Hovestadt V, Khuong-Quang D-A, Jones David TW, Konermann C, Pfaff E, Tönjes M, Sill M, Bender S et al (2012) Hotspot mutations in $\mathrm{H} 3 \mathrm{~F} 3 \mathrm{~A}$ and $\mathrm{IDH} 1$ define distinct epigenetic and biological subgroups of glioblastoma. Cancer Cell 22:425-437

56. Suzuki H, Aoki K, Chiba K, Sato Y, Shiozawa Y, Shiraishi Y, Shimamura T, Niida A, Motomura K, Ohka F et al (2015) Mutational landscape and clonal architecture in grade II and III gliomas. Nat Genet 47:458-468

57. Thalheim T, Hopp L, Binder H, Aust G, Galle J (2018) On the cooperation between epigenetics and transcription factor networks in the specification of tissue stem cells. Epigenomes 2:20

58. Toronen P, Ojala P, Marttinen P, Holm L (2009) Robust extraction of functional signals from gene set analysis using a generalized threshold free scoring function. BMC Bioinformatics 10:307

59. Venteicher AS, Tirosh I, Hebert C, Yizhak K, Neftel C, Filbin MG, Hovestadt V, Escalante LE, Shaw ML, Rodman C et al (2017) Decoupling genetics, lineages, and microenvironment in IDH-mutant gliomas by single-cell RNAseq. Science 355(6332)

60. Verhaak RGW, Hoadley KA, Purdom E, Wang V, Qi Y, Wilkerson MD, Miller CR, Ding L, Golub T, Mesirov JP et al (2010) Integrated genomic analysis identifies clinically relevant subtypes of glioblastoma characterized by abnormalities in PDGFRA, IDH1, EGFR, and NF1. Cancer Cell 17:98-110

61. von Schimmelmann M, Feinberg PA, Sullivan JM, Ku SM, Badimon A, Duff MK, Wang Z, Lachmann A, Dewell S, Ma'ayan A et al (2016) Polycomb repressive complex 2 (PRC2) silences genes responsible for neurodegeneration. Nat Neurosci 19:1321

62. Wang Q, Hu B, Hu X, Kim H, Squatrito M, Scarpace L, de Carvalho AC, Lyu S, Li P, Li Y et al (2017) Tumor Evolution of Glioma-Intrinsic Gene Expression Subtypes Associates with Immunological Changes in the Microenvironment. Cancer Cell 32:42-56 e46

63. Ward PS, Cross JR, Lu C, Weigert O, Abel-Wahab O, Levine RL, Weinstock DM, Sharp KA, Thompson CB (2012) Identification of additional IDH mutations associated with oncometabolite $\mathrm{R}(-)$-2-hydroxyglutarate production. Oncogene 31:2491-2498 
64. Watanabe T, Nobusawa S, Kleihues P, Ohgaki H (2009) IDH1 mutations are early events in the development of Astrocytomas and Oligodendrogliomas. Am J Pathol 174:1149-1153

65. Weller M, Weber R, Willscher E, Riehmer V, Hentschel B, Kreuz M, Felsberg J, Beyer U, Löffler-Wirth H, Kaulich K et al (2015) Molecular classification of diffuse cerebral WHO grade II/III gliomas using genome- and transcriptomewide profiling improves stratification of prognostically distinct patient groups. Acta Neuropathol 129(5):679-93

66. Weller M, Wick W, Aldape K, Brada M, Berger M, Pfister SM, Nishikawa R, Rosenthal M, Wen PY, Stupp R, Reifenberger G (2015) Glioma. In: Nature reviews Disease primers, vol 1, p 1501715017

67. Wirth $H$, Löffler $M$, von Bergen M, Binder H (2011) Expression cartography of human tissues using self organizing maps. BMC Bioinformatics 12:306

68. Wirth H, von Bergen M, Binder H (2012) Mining SOM expression portraits: Feature selection and integrating concepts of molecular function. BioData Mining 5:18

69. Xie W, Kagiampakis I, Pan L, Zhang YW, Murphy L, Tao Y, Kong X, Kang B, Xia L, Carvalho FLF et al (2018) DNA Methylation Patterns Separate Senescence from Transformation Potential and Indicate Cancer Risk. Cancer Cell 33:309-321 e305

70. Yang XW (2016) Life and death rest on a bivalent chromatin state Nat Neurosci 19:1271

71. Yao C, Li H, Shen X, He Z, He L, Guo Z (2012) Reproducibility and concordance of differential DNA methylation and gene expression in Cancer. PLoS One 7:e29686

72. Yao L, Shen H, Laird PW, Farnham PJ, Berman BP (2015) Inferring regulatory element landscapes and transcription factor networks from cancer methylomes. Genome Biol 16:105-105

73. Yawata T, Nakai E, Park KC, Chihara T, Kumazawa A, Toyonaga S, Masahira T, Nakabayashi H, Kaji T, Shimizu K (2010) Enhanced expression of cancer testis antigen genes in glioma stem cells. Mol Carcinog 49:532-544

Ready to submit your research? Choose BMC and benefit from:

- fast, convenient online submission

- thorough peer review by experienced researchers in your field

- rapid publication on acceptance

- support for research data, including large and complex data types

- gold Open Access which fosters wider collaboration and increased citations

- maximum visibility for your research: over $100 \mathrm{M}$ website views per year

At $\mathrm{BMC}$, research is always in progress.

Learn more biomedcentral.com/submissions 